\title{
Leucaemia cutis bei akuter Monozytenleukämie
}

\author{
G. Wagner
}

\author{
Cutaneous Manifestation in Acute Monocytic Leukemia
}

\section{Zusammenfassung}

Die systemischen Leukämien können sich am Hautorgan in klinisch-morphologisch unterschiedlichen Formen manifestieren, die bei histopathologischem Nachweis einer leukämischen Infiltration als Leucaemia cutis oder auch als spezifische Hautveränderungen einer zugrunde liegenden Leukämie bezeichnet werden. Hiervon abzugrenzen sind unspezifische kutane Begleitreaktionen, die zumeist Ausdruck einer gestörten Hämatopoese sind oder als kutane Paraneoplasien eingeordnet werden können. Zur typischen klinischen Morphologie der spezifischen Hautveränderungen zählen umschriebene oder flächenhafte Erytheme, Papeln, Knoten, Plaques und Ulzera. Am Beispiel zweier Patienten mit akuter Monozytenleukämie werden die klinische Variabilität der spezifischen Läsionen und ihre jeweiligen Differentialdiagnosen vorgestellt. Bei beiden Patienten war die akute Monozytenleukämie erst im Verlauf der dermatologischen Diagnostik aufgedeckt worden, was einerseits die Bedeutung der spezifischen dermatologischen Veränderungen unterstreicht, andererseits aber auch die Grenzen der klinischen Morphologie aufzeigt, da ebenfalls bei beiden Patienten zunächst andere Dermatosen favorisiert worden waren.

\section{Abstract}

Systemic leukemia can show different clinical-morphological manifestations in skin. These are described as leukemia cutis or as a specific leukemia-related skin lesion according to specific histopathological findings. They have to be distinguished from unspecific cutaneous reactions due to disturbed haematopoesis or, as a result of other tumours - from so called cutaneous neoplasia. Typical clinical-morphological skin reactions in systemic leukemia are circumscribed or extensive erythema, papules, nodules, plaque and ulceration. In this case report two patients with acute monocytic leukemia with their clinical variability of the specific skin lesions as well as the differential diagnosis are presented. In both patients monocytic leukemia was detected during diagnostic procedure although other skin diseases were favoured prior to diagnosing the leukemia. On the one hand this emphasizes the significance of the specific skin lesions and shows the limitation of clinical morphology, on the other hand.

\section{Einleitung}

Die verschiedenen Formen der Leukämien werden als maligne Neoplasien des hämatopoetischen Systems definiert, wobei es nach einer Generalisation im Knochenmark und einer Ausschwemmung der malignen Zellreihen in das periphere Blut zu einem Befall extramedullärer Organsysteme einschließlich der
Haut kommen kann [1]. Auf Grund des biologischen Verhaltens und des jeweils vorliegenden Differenzierungsgrades der neoplastischen Zellen werden akute und chronisch verlaufende Leukämieformen voneinander abgegrenzt. Die akute lymphatische Leukämie, deren Inzidenz mit 1:100000 Einwohner pro Jahr angegeben wird, zeichnet sich durch eine biphasische Häufigkeitsverteilung aus mit einem ersten Häufigkeitsgipfel bei Kindern im 
Alter zwischen zwei und vier Jahren und einem zweiten Gipfel in der siebenten Lebensdekade. Mit 30\% ist die akute lymphatische Leukämie die häufigste Krebserkrankung der Kinder überhaupt [2]. Die akute myeloische Leukämie zeigt hingegen eine grundsätzlich andere Häufigkeitsverteilung. Während sie bei Kindern, Jugendlichen und Erwachsenen bis zum 30. Lebensjahr nur selten beobachtet wird, steigt ihre Inzidenz anschließend kontinuierlich an. Bei den über 70-Jährigen wird mit einer jährlichen Häufigkeit von zehn Neuerkrankungen pro 100000 Menschen gerechnet [3]. Die Subtypisierung der akuten Leukämien erfolgt auf der Grundlage der Empfehlungen der French-American-British Cooperative Group (FAB) unter Berücksichtigung morphologischer, zytogenetischer und immunphänotypischer Merkmale [4]. Dabei wird die akute lymphatische Leukämie in die Typen $\mathrm{L}_{1}-\mathrm{L}_{3}$ und die akute myeloische Leukämie in die Typen $\mathrm{M}_{1}-\mathrm{M}_{7}$ eingeteilt. Die akute Monozytenleukämie, die bei den beiden hier vorgestellten Patienten diagnostiziert werden konnte, entspricht dem Typ $\mathrm{M}_{5}$ der akuten myeloischen Leukämie. Innerhalb der Gruppe der chronisch verlaufenden Leukämien ist die chronische lymphatische Leukämie mit 30\% die häufigste Leukämieform der westlichen Welt. Auch ihre Inzidenz ist altersabhängig, wobei die Erkrankung in den ersten vier Lebensdekaden selten ist, um dann bei den 50-Jährigen eine Inzidenz von $5: 100000$ und bei den 80-Jährigen eine solche von 30:100 000 zu erreichen [5]. Die chronische myeloische Leukämie, deren Inzidenz mit jährlich zwei Neuerkrankungen pro 100000 Einwohner angegeben wird, zeigt einen Altersgipfel in der fünften Lebensdekade, wobei etwa $20 \%$ aller Leukämien der chronischen myeloischen Form zugerechnet werden können [6].

Die klinische Symptomatik der Leukämien hängt von ihrer Verlaufsform ab. Bei den akuten Leukämien wird das klinische Bild durch eine häufig fulminant zunehmende Knochenmarkinsuffizienz geprägt. Dabei ist ein schweres Krankheitsbild mit hohem Fieber, Infektionen der oberen Luftwege, gastrointestinalen Symptomen, Anorexie, Knochen- und Gelenkschmerzen sowie Blutungen der Haut und Schleimhäute typisch [2]. Im Gegensatz hierzu werden die chronischen Leukämien häufig zufällig entdeckt, z.B. durch routinemäßige Blutbilduntersuchungen oder durch asymptomatische Splenomegalien bei Oberbauchsonographien. Allgemeines Unwohlsein, zunehmende Schwäche, schleichender Gewichtsverlust, subfebrile Temperaturen und uncharakteristische Oberbauchbeschwerden sind klinische Symptome der chronischen Leukämieformen [7].

Die diagnostische Einordnung der spezifischen oder unspezifischen Hautveränderungen einer Leukämie dürfte in den meisten Fällen zunächst nur als Möglichkeit im Rahmen differentialdiagnostischer Überlegungen erfolgen. Die Kenntnis der aufgeführten epidemiologischen Daten und der allgemein-klinischen Symptome der Leukämien kann dabei im Einzelfall hilfreich sein, um eine weiterführende Diagnostik zu veranlassen, wie sich dies auch am Beispiel der folgenden Kasuistiken zeigen lässt.

\section{Kasuistiken}

\section{Kasuistik I: Patient LK, 69 Jahre \\ Anamnese}

Vier Wochen vor der stationären Aufnahme hatte der Patient erstmals juckende Hautveränderungen an den Unterschenkeln bemerkt, die anschließend generalisierten. Im Zusammenhang mit einem femoro-poplitealen Bybass bei bekannter AVK war der Patient seit einem Jahr marcumarisiert. Die Einnahme weiterer Medikamente verneinte er. Die Einweisung des Patienten erfolgte durch eine niedergelassene Dermatologin unter der Verdachtsdiagnose eines papulösen Arzneimittelexanthems. Das Allgemeinbefinden war bei stationärer Aufnahme in keiner Weise eingeschränkt.

\section{Dermatologischer Aufnahmebefund}

An den oberen und unteren Extremitäten sowie am Stamm in exanthematischer Ausbreitung einzeln stehende oder auch konfluierende, linsen- bis halbkirschkerngroße, derbe, hautfarbene oder rötlich tingierte Papeln und Knoten (Abb.1,2). Im Bereich der Mundschleimhäute einzelne linsengroße, geringfügig infil-

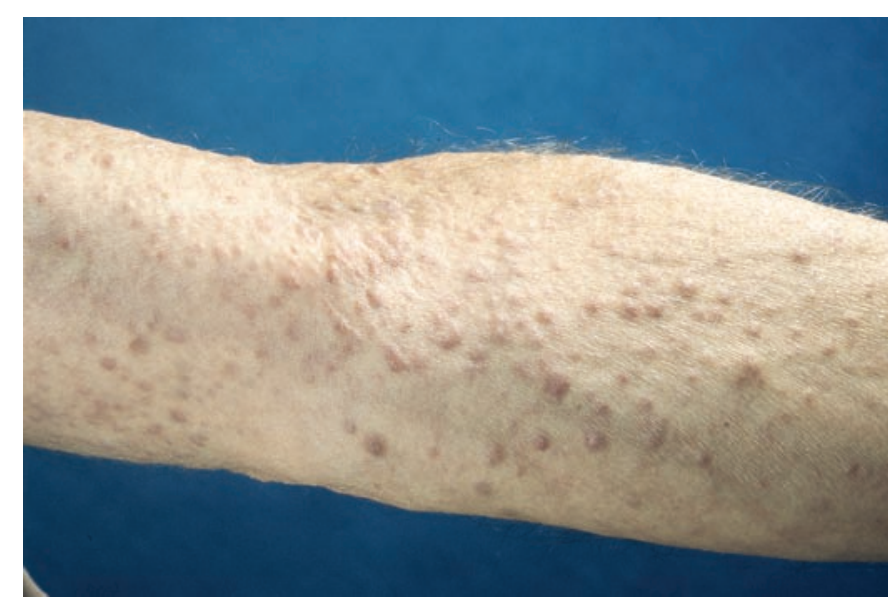

Abb. 1 Pat. I: Disseminiert stehende Knoten Beugeseiten linker Arm.

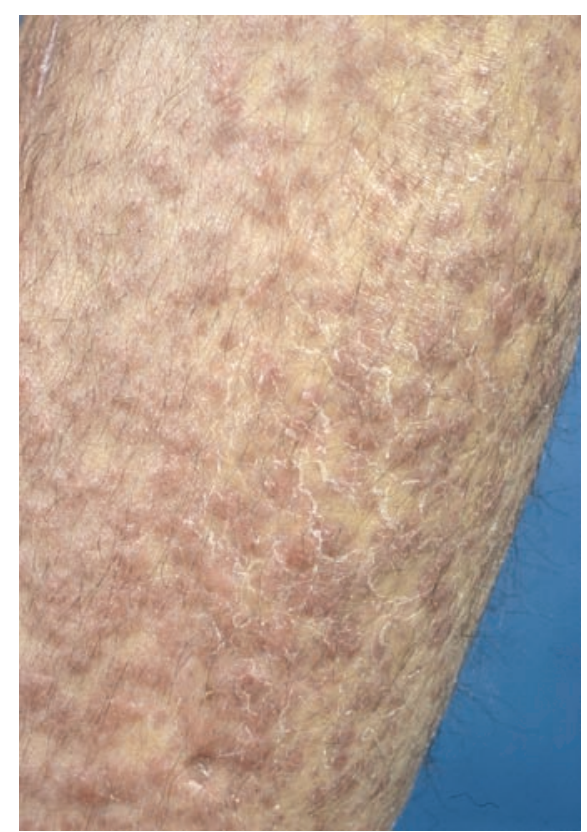

Abb. 2 Pat. I: Konfluierende, plaqueförmige Knoten Streckseite linker Unterschenkel. 
trierte Erytheme. Die allgemein-körperliche Untersuchung war unauffällig. Insbesondere Lymphknoten konnten nicht getastet werden.

\section{Befunde diagnostischer Untersuchungen}

Histopathologische Befunde

Biopsie einer Papel linker Unterarm: Bei unauffälliger Epidermis monomorphe Population atypischer monozytärer Zellen mit ovalen Zellkernen und breitem eosinophilen Zytoplasma in der oberen und mittleren Dermis. Immunhistochemisch exprimieren die atypischen Zellen CD43, Lysozym, CD68 und fokal Chloracetatesterase. Diagnose: Leucaemia cutis bei myelomonozytärer Leukämie.

Knochenmarkbiopsie Beckenkamm: Ausgedehnte, etwa 80\% der Markfläche einnehmende Infiltrate, bestehend aus Blasten mit polymorphen, teilweise auch gelappten Kernen. Helles Kernchromatin, zum Teil deutliche Nukleolen. Hämatopoese stark vermindert. Immunhistochemisch positive Expression Monozytenmarker Ki-M1p und Lysozym, negativ hingegen bei myeloischem Marker Ki-My2, B-Zell-Marker CD79a und T-Zell-Marker CD3. Diagnose: Akute Monozytenleukämie FAB M5.

Laborbefunde

Blutbild: Leukozyten 6,9 (NW: 4 - 10,5/nl), Erythrozyten 4,85

(NW: 3,5 - 5,8/pl), Hämoglobin 12.9 (NW: 14,0 - 18,0 g/dl), Hämatokrit 38.8 (NW: 38 - $51 \mathrm{cl} / \mathrm{l}$ ), MCV 79.8 (NW: 83 - 99 fl), HbE 26.6 (NW: 27 - 34 pg/Ery), RDW 17.1 (NW: 11 - 16\% VC), Thrombozyten 115 (NW: $130-450 / \mathrm{nl}$ ).

Differentialblutbild: Myelozyten 1 (NW: 0\%), Stabkernige 8 (NW: $0-6 \%$ ), Segmentkernige 22 (NW: $45-85 \%$ ), Basophile 1 (NW: 0 - 3\%), Monozyten 29 (NW: 1 - 11\%), Lymphozyten 34 (NW: $10-50 \%$ ), atypische Zellen 5 (NW: $0-3 \%$ ). BSG n.W. 32/66 (NW: 10 - $20 \mathrm{~mm}$ ), CRP 49 (NW: 3 - $15 \mathrm{mg} / \mathrm{l}$ ), LDH 733 (NW: $240-480 \mathrm{U} / 1$ ).

Ohne pathologischen Befund: Nierenpflichtige Substanzen, Lebertransaminasen, Bilirubin, Elektrolyte, Eiweißelektrophorese und Urinstatus.

\section{Ergänzende Untersuchungen}

Rö.-Thorax und Oberbauchsonographie ohne pathologische Befunde. Sonographie Leisten und Achseln mit Nachweis einzelner bis zu einem cm durchmessender, sonomorphologisch unauffälliger Lymphknoten. CT Thorax und Abdomen: vereinzelt sehr kleine axilläre, mediastinale und paraaortale Lymphknoten. Keine größeren Lymphknotenpakete, intrapulmonal keine pathogenen Befunde, keine Hepatosplenomegalie oder andere pathologische Befunde intraabdominell.

\section{Therapie und Verlauf}

Auf Grund der erhobenen Befunde wurde bei dem Patienten die Diagnose einer Leucaemia cutis bei akuter Monozytenleukämie gestellt. Zur weiteren Behandlung erfolgte die Verlegung in eine hämatologisch-onkologische Klinik. Unter der dort durchgeführten Chemotherapie mit Daunorubicin und Cytarabin kam es zu einer vollständigen Remission, die durch eine komplette Rückbildung des dermatologischen Befundes und eine Kontrollbiop- sie des Knochenmarks dokumentiert werden konnte. Der weitere Verlauf bleibt abzuwarten.

\section{Kasuistik II: Patientin BC, 72 Jahre Anamnese}

Die zum Zeitpunkt der stationären Aufnahme bereits schwer kranke Patientin war unter der Diagnose eines fieberhaften Infektes in die Medizinische Klinik des Hauses eingewiesen worden. Seit einer Woche bestanden Temperaturen bis $40^{\circ} \mathrm{C}$, Husten sowie schmerzhafte Erosionen der Mundschleimhaut. Unmittelbar vor der Einweisung waren eine Makrohämaturie und erstmals auch nicht juckende Hautveränderungen an den Extremitäten beobachtet worden.

\section{Dermatologischer Aufnahmebefund}

Unter Betonung der Streckseiten der Unterschenkel an den oberen und unteren Extremitäten münz- bis handtellergroße, auch konfluierende, unscharf begrenzte, teilweise deutlich infiltrierte, druckdolente Erytheme (Abb.3,4). Im Bereich der Mundschleimhäute linsen- bis fingernagelgroße, schmierig belegte Erosionen.

\section{Befunde diagnostischer Untersuchungen}

Histopathologische Befunde

Biopsie linker Unterschenkel: Bei durchgehend erhaltener Epidermis hochgradig ödematös aufgelockertes Korium. Perivaskulär finden sich hier mononukleäre Infiltrate, die eine mittlere Proliferationsaktivität von 20\% mit Ki-67 aufweisen. Sie exprimieren Ki-M1P zu über 90\%. Diagnose: Neoplastische Monozyteninfiltration. Befund passt in erster Linie zu einer akuten Monozytenleukämie (FAB M5). Gelegentlich treten derartige Hautbefunde bereits in der myelodysplastischen Vorphase einer Monozytenleukämie auf.

Knochenmarkbiopsie Beckenkamm: Normozelluläre Hämatopoese. Quantitativ etwas vermehrte, deutlich linksverschobene Megakaryopoese. Linksverschobene Erythropoese mit Reifungsstö-

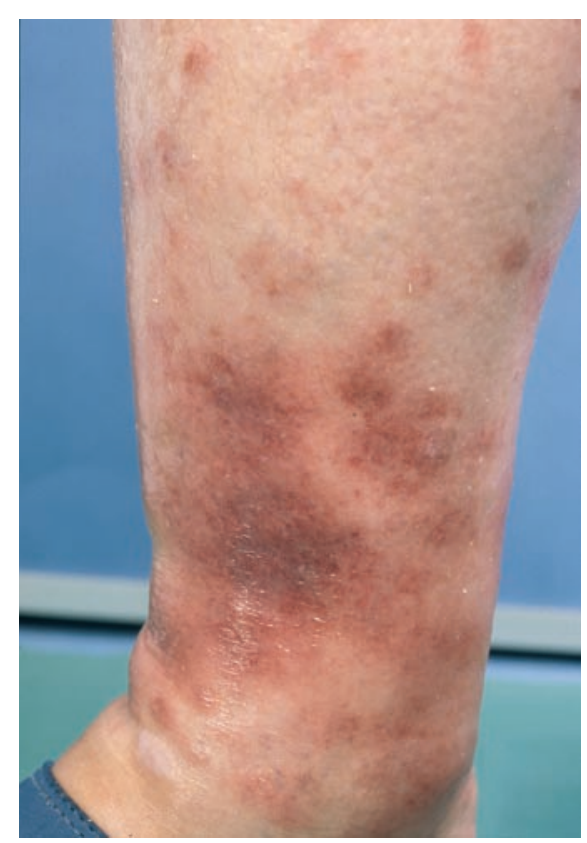

Abb. 3 Pat. II: Flächenhaft infiltrierte Erytheme distaler Unterschenkel rechts. 


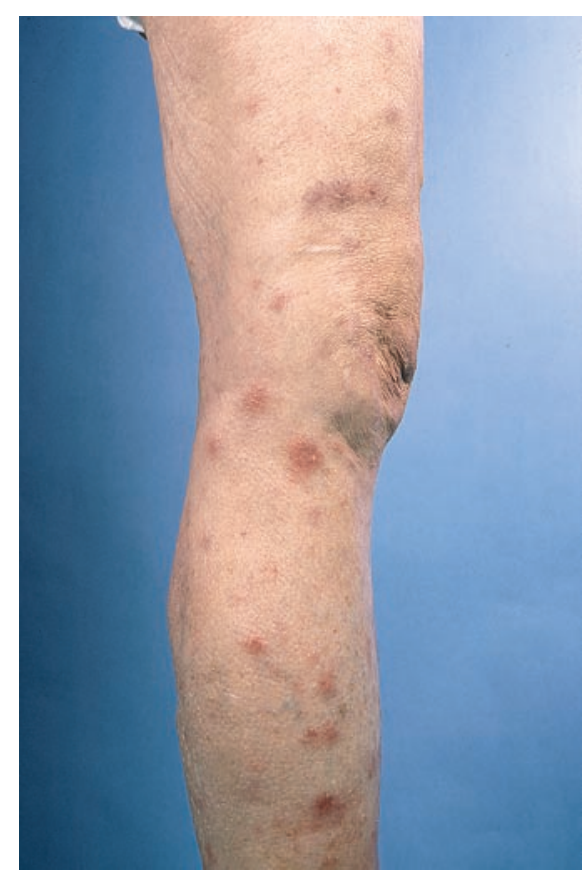

Abb. 4 Pat. II:

Einzeln stehende, infiltrierte Erytheme rechte untere Extremität.

rung. Granulopoese ganz erheblich linksverschoben und hochgradig reifungsgestört. Nur ganz vereinzelt segment- oder stabkernige Granulozyten. Vorherrschen von myeloischen blastären Vorstufen. Blastenanteil ca. 30\%. Diagnose: Myelodysplastisches Syndrom mit Reifungsstörung aller Zellreihen und Blastenexzess.

\section{Laborbefunde}

Blutbild: Leukozyten 1,9 (NW: 4 - 10,5/nl), Erythrozyten 2,90 (/NW: 3,5 - 5,8/pl), Hämoglobin 8,3 (NW: 12,3 - 15,3 g/dl), Hämatokrit 25,2 (NW: 38 - $51 \mathrm{cl} / \mathrm{l}$ ), MCV 86,9 (NW: 83 - 99 fl), HbE 28,7 (NW: 27 - 34 pg/Ery), RDW 28,1 (NW: 11 - 16\% VC), Thrombozyten 65 (NW: $130-450 / \mathrm{nl}$ ).

Differentialblutbild: Neutrophile 8 (NW: 45 - 85\%), Eosinophile 0 (NW: 0 - 6\%), Basophile 1 (NW: 0 - 2\%), Monozyten 48 (NW: 0 $11 \%$ ), Lymphozyten 43 (NW: 10 - 50\%). BSG n. W. 56/98 (NW: 10 - 20 mm), CRP 81 (NW: 3 - 15 mg/l). Protein- und Hämaturie.

Ohne pathologische Befunde: Nierenpflichtige Substanzen, Lebertransaminasen, LDH, CK, Bilirubin und Elektrolyte.

Ergänzende Untersuchungen

Rö.-Thorax: Ausgedehnte Oberlappenpneumonie beidseits mit Begleiterguss. Flaues Infiltrat rechts, offenbar im Mittellappen gelegen.Oberbauchsonographie: Splenomegalie $(14,5 \times 6,5 \mathrm{~cm})$, leichte Hepatomegalie.

\section{Therapie und Verlauf}

Trotz intensivmedizinischer Betreuung, bei der die Behandlung der Pneumonie im Vordergrund stand, verschlechterte sich der Zustand der Patientin kontinuierlich. Gleichzeitig entwickelte sich eine Leukozytose von maximal 90/nl mit 95\% Leukämiezellen sowie einer Thrombozytopenie von 5/nl. Die Patientin verstarb an der nicht beherrschbaren Pneumonie im akuten HerzKreislaufversagen.
Diskussion

Die Leukämien können sich durch eine sehr unterschiedliche kutane Symptomatik auszeichnen, wobei spezifische und unspezifische Hautveränderungen voneinander abgegenzt werden. Als spezifisch gelten Hautveränderungen, die sich histopathologisch durch ein leukämisches Infiltrat auszeichnen. Unabhängig von der jeweils vorliegenden klinischen Morphologie werden diese Hautveränderungen zusammengefasst auch als Leucaemia cutis bezeichnet. Die unspezifischen Hautveränderungen entwickeln sich hingegen als Folge einer gestörten Hämatopoese oder stellen paraneoplastische Reaktionen dar [8]. Einzelne Hautveränderungen, z.B. hämorrhagische Phänomene oder Ulzera, können sowohl Ausdruck einer spezifischen als auch einer unspezifischen Genese sein. Die spezifischen Manifestationen der Leukämien sind insgesamt seltene Ereignisse, wenngleich ihre Häufigkeit bei den verschiedenen Leukämieformen deutliche Unterschiede aufweisen kann. Dabei sind die in der Literatur gemachten Angaben zur Häufigkeit der Leucaemia cutis untereinander nicht immer vergleichbar, da die Terminologie und die Klassifikation der Leukämien in der Vergangenheit nicht einheitlich benutzt worden sind. Unbestritten werden spezifische Hautveränderungen in der Praxis am häufigsten bei der chronischen lymphatischen Leukämie beobachtet, ein Umstand, der allerdings darauf zurückzuführen sein dürfte, dass die chronische lymphatische Leukämie selbst die häufigste Leukämieform darstellt und darüber hinaus einen meist langfristigen Verlauf zeigt $[9,10]$. Die entsprechende Häufigkeit wird in der Literatur mit 8 - 27\% angegeben $[11,12]$. Betrachtet man die Häufigkeit der Leucaemia cutis bezogen auf die absolute Zahl der einzelnen Leukämieformen, finden sich spezifische Hautveränderungen relativ am häufigsten bei den akuten myeloischen Leukämien $[9,13]$. In größeren Studien fanden sich über $40 \%$ der spezifischen Hautveränderungen bei Patienten mit akuten myeloischen Leukämien [14,15]. Innerhalb dieser Gruppe ist wiederum die akute Monozytenleukämie mit 25-33\% am häufigsten mit einer Leucaemia cutis verbunden [14-17]. Interessanterweise finden sich bei den akuten myeloischen Leukämien auch überproportional häufig leukämische Lungeninfiltrate. Eine Erklärung hierfür ist möglicherweise die für reife myeloische Zellen typische Expression von Adhäsionsmolekülen [18]. Diese biologische Eigenschaft könnte dann auch als Grund für die Häufigkeit der Leucaemia cutis bei den akuten myeloischen Leukämien herangezogen werden. Im Gegensatz zu den genannten Leukämieformen sind spezifische Hautveränderungen bei der akuten lymphatischen und der chronischen myeloischen Leukämie deutlich seltener. Die Häufigkeitsangaben liegen hier zwischen 2 und 3\% [19,20].

Die spezifischen Hautveränderungen der Leukämien zeichnen sich durch eine ausgeprägte klinische Polymorphie aus. Dabei können alle Variationen der Leucaemia cutis bei jeder Leukämieform auftreten, so dass auf Grund der im Einzelfall vorliegenden klinischen Morphologie nicht auf die Art der zu Grunde liegenden Leukämie geschlossen werden kann [10]. Allerdings können einzelne Formen der Leucaemia cutis bei bestimmten Leukämien deutlich häufiger beobachtet werden, wie z.B. die Gingivahyperplasie, die Makrulie, bei der akuten Monozytenleukämie [21]. Darüber hinaus können die spezifischen Hautveränderungen in einer einzelnen Form oder in jeder beliebigen Kombination der Morphen auftreten. In den meisten Fällen manifestiert sich eine 
Leucaemia cutis erst im Verlauf einer bereits bekannten Leukämie. Seltener können die spezifischen Hautveränderungen parallel mit der internistischen Symptomatik auftreten oder dieser in Einzelfällen als so genannte aleukämische Leucaemia cutis sogar vorausgehen [22 - 24]. Die häufigsten Manifestationsformen der Leucaemia cutis sind Papeln, Knoten oder plaqueförmige Infiltrationen, die exanthematisch oder in Form weniger Einzelherde auftreten können. Die Häufigkeit der knotigen Morphen wird in einzelnen Untersuchungen mit bis zu 60\% angegeben [10]. In Abhängigkeit von ihrer Größe und Lokalisation innerhalb der kutanen Strukturen werden hautfarbene, gelbliche, erythematöse, livide oder auch braun pigmentierte Knoten beobachtet $[21,25,26]$. Zu den bevorzugt betroffenen Arealen zählen in der Reihenfolge ihrer Häufigkeit der Stamm, die Extremitäten, der Kopf und der Nacken [10]. Der Befall des Gesichtes mit monströsen Knoten oder Plaques wird dabei als Facies leontina bezeichnet [21]. Als eine weitere Sonderform knotiger Manifestationen der Leucaemia cutis wird das sogenannte Chlorom beschrieben. Dieser seltene Tumor, der durch leukämische Infiltrationen der Perioststrukturen der Orbita oder anderer Knochen entsteht, ist gekennzeichnet durch eine grünliche Verfärbung, verursacht durch eine hohe Gewebekonzentration von Myeloperoxidase [27]. Für alle knotigen Strukturen typisch ist ein derber Palpationsbefund. Vereinzelt können größere Knoten sekundär ulzerieren $[21,25,26,28]$. Umschriebene, unscharf begrenzte Erytheme mit oder ohne Schuppung, Ekchymosen, die sowohl Ausdruck einer leukämischen Infiltration als auch Folge einer Thrombozytopenie sein können, sowie Erythrodermien stellen weitere, jedoch seltener vorkommende Manifestationen der Leucaemia cutis dar. Dabei variieren die Häufigkeitsangaben zwischen 19\% für Erytheme, 17\% für Ekchymosen und weniger als 5\% für Erythrodermien [10]. Letztere werden in ihrer klinischen Ausprägung als Erytheme ohne weitere morphologische Kriterien oder als exsudativ-ekzematöse Formen beschrieben [29]. Als gelegentlich beobachtete Manifestation einer Leucaemia cutis gelten primär auftretende, einzeln oder disseminiert vorkommende Ulzera, die mit einem maximal beschriebenen Durchmesser von bis zu $10 \mathrm{~cm}$ eine beachtliche Größe erreichen können [30,31]. Die ebenfalls nur selten vorkommenden Blasen vervollständigen das klinische Spektrum der Leucaemia cutis [10]. Neben den beschriebenen Manifestationen der Leucaemia cutis am äußeren Integument findet sich eine Vielzahl spezifischer Hautveränderungen auch im Bereich der Mundschleimhäute. Dabei steht die Gingivahyperplasie mit einer Häufigkeit von 3 - 5\% zahlenmäßig im Vordergrund [32]. Klinisch-morphologisch finden sich ödematös-knotige oder plattenförmige Infiltrationen, die die Zähne überwuchern können. Nekrosen, tiefgreifende Ulzera und Zahnverlust sind mögliche Folgen der leukämischen Infiltrationen.

Von den unterschiedlichen Erscheinungsformen der Leucaemia cutis sind die deutlich häufiger vorkommenden, unspezifischen Hautveränderungen der verschiedenen Leukämien abzugrenzen, wobei spezifische und unspezifische Hautveränderungen auch gleichzeitig auftreten können. Bezogen auf die gesamte Gruppe der Leukämien finden sich bei 25 - 40\% der betroffenen Patienten unspezifische Hautveränderungen [33]. Allerdings gibt es auch hier wieder teilweise deutliche Unterschiede zwischen den einzelnen Leukämieformen [34]. So wird z B. die Häufigkeit der unspezifischen Hautveränderungen bei der Monozytenleukämie vereinzelt mit bis zu 80\% angegeben [8]. Die unspezifi- schen Hautveränderungen entwickeln sich auf der Grundlage der gestörten Hämatopoese oder werden als paraneoplastische Reaktionen verstanden. Die besonders bei den akuten Leukämien häufig vorkommende Thrombozytopenie ist dabei für verschiedene hämorrhagische Phänomene verantwortlich, z.B. für Petechien, Ekchymosen oder Sugillationen. Hingegen dürfte die ebenfalls zu beobachtende palpable Purpura typischerweise auf eine paraneoplastische Vasculitis allergica zurückzuführen sein $[34,35]$. Die gestörte Granulozytopoese wird für die gehäuft vorkommenden opportunistischen Infektionen verantwortlich gemacht. Für Leukämien typisch sind dabei chronisch-persistierende Herpes simplex-Eruptionen, der Herpes zoster generalisatus, chronische Follikulitiden, Abszessbildungen und klinischmorphologisch ungewöhnliche Mykosen [10,21,28,34].

Zu den paraneoplastischen Reaktionen zählen der generalisierte, häufig therapieresistente Pruritus, urtikarielle, ekzematoide oder makulopapulöse Exantheme sowie das Erythema nodosum und das Erythema exsudativum multiforme [27]. Auch das Sweet-Syndrom und das Pyoderma gangraenosum können als unspezifische Hautveränderungen im Rahmen einer Leukämie auftreten. Bei einer umfangreichen Literaturstudie zum SweetSyndrom fanden sich bei $20 \%$ der Patienten assoziierte Malignome, von denen wiederum fast $40 \%$ an einer akuten myeloischen Leukämie erkrankt waren [36]. Das mit einem Malignom verbundene Sweet-Syndrom kann dabei klinisch ungewöhnliche, bullöse oder ulzerierende Formen aufweisen. Die Assoziation zwischen einem Pyoderma gangraenosum und den verschiedenen Leukämieformen ist ebenfalls gehäuft beobachtet worden. Auch hierbei gilt, dass klinisch ungewöhnliche Manifestationsformen eines Pyoderma gangraenosum als Hinweis für die Existenz einer malignen Grunderkrankung gelten müssen [27]. Auf Grund ihrer ungewöhnlichen Häufigkeit können auch Arzneimittelexanthme als unspezifische Hautveränderungen der Leukämien eingeordnet werden. Bei einzelnen Untersuchungen konnten bei mehr als $50 \%$ der Patienten Arzneimittelexantheme beobachtet werden, die durch chemisch völlig unterschiedliche Arzneimittelgruppen ausgelöst worden waren [38]. Als besondere Form einer Arzneimittelreaktion wird die sogenannte „eruption of lymphocyte recovery" von den herkömmlichen Arzneimittelexanthemen abgegrenzt. Dabei entwickelt sich meist während der Induktionsphase einer Chemotherapie ein makulopapulöses Exanthem, das typischerweise unmittelbar nach dem Tiefpunkt der Leukozytopenie auftritt [39]. Die differentialdiagnostischen Überlegungen zu den verschiedenen Formen der Leucaemia cutis sind von der jeweils vorliegenden klinischen Morphologie abhängig. Darüber hinaus ist von entscheidender Bedeutung, ob bei einem betroffenen Patienten eine Leukämie bereits bekannt ist oder ob in der möglicherweise initialen Phase der Erkrankung eine allgemeine Symptomatik besteht, die auf eine Leukämie hindeuten könnte. So die eine oder die andere Situation gegeben ist, wird man insbesondere beim Auftreten der doch relativ charakteristischen papulösen Morphen frühzeitig an eine Leucaemia cutis denken müssen. Ist hingegen eine Leukämie nicht bekannt, fehlen richtungsweisende Symptome einschließlich der entsprechenden Blutbildveränderungen oder wird eine allgemeine Krankheitssymptomatik zunächst als Ausdruck einer banalen Infektion gewertet, dürfte die Leucaemia cutis im Allgemeinen eine Zufallsdiagnose sein, die erst durch eine histopathologische Untersuchung der vorliegenden Hautverän- 
derungen aufgedeckt wird. Eine solche Situation war auch bei den hier vorgestellten Patienten gegeben. Bei dem ersten Patienten hatte sich ein papulöses Exanthem entwickelt, ohne dass Einschränkungen des Allgemeinbefindens erfragt werden konnten. Auch eine Leukämie war zum Zeitpunkt der stationären Aufnahme nicht bekannt gewesen. Auf Grund des klinischen Befundes hatte die einweisende Dermatologin die Verdachtsdiagnose eines papulösen Arzneimittelexanthems gestellt, während unsererseits an eine Sarkoidose gedacht wurde. Beide Differentialdiagnosen werden in der Literatur beschrieben $[8,21,24]$. Die stationäre Einweisung der zweiten Patientin in die Innere Klinik des Hauses erfolgte unter der Diagnose eines fieberhaften Infektes, wobei die Rö.-Aufnahme der Thoraxorgane eine beidseitige Pneumonie zeigte. Die erst sehr kurzfristig bestehenden Hautveränderungen wurden zunächst als Erythema nodosum eingeordnet. Erst die nachgewiesene Panzytopenie und die Therapieresistenz der Pneumonie waren Anlass zur Durchführung einer Biopsie, deren histopathologische Beurteilung dann zur Diagnose einer Leucaemia cutis führte. Dabei zählen sowohl das Erythema nodosum als auch das Erythema exsudativum multiforme zu wiederholt genannten Differentialdiagnosen der Leucaemia cutis $[8,10,28]$. Die weiteren im Einzelfall zu berücksichtigen Differentialdiagnosen umfassen maligne Lymphome und Pseudolymphome, kutane Metastasen viszeraler Karzinome, makulöse oder papulöse syphilitische Exantheme sowie atypische Formen der Pityriasis rosea. Die leukämischen Manifestationen im Bereich der Mundschleimhäute sind insbesondere von der Angina PlautVincent abzugrenzen $[8,21,24]$.

Das Spektrum der möglichen Differentialdiagnosen und die Besonderheiten im klinischen Verlauf der verschiedenen Leukämieformen unterstreichen die Probleme bei der diagnostischen Einordnung einer Leucaemia cutis. Auf Grund der phänomenologischen Ähnlichkeiten mit zahlreichen Dermatosen wird die Diagnose einer Leucaemia cutis letztendlich immer histopathologisch gestellt werden müssen.

\section{Literatur}

${ }^{1}$ Wilms K. Akute Leukämien. In: Huhn W, Wilms K (Hrsg). Internistische Onkologie. Stuttgart: Thieme, 1994

${ }^{2}$ Creutzig U, Schrappe M. Akute Leukämien im Kindesalter. Internist 1996; 37: $982-993$

${ }^{3}$ Copplestone JA, Prentice AG. Acute myeloblastic leukaemia in the elderly. Leuk Res 1988; 8: 617-625

${ }^{4}$ French-American-British (FAB) Cooperative Group, Bennet JM, Catovsky D, Daniel MT. Proposals for the classification of the acute leukaemias. Brit J Haematol 1976; 33: 489-496

${ }^{5}$ Borchmann P, Diehl V, Engert A. Die chronisch lymphatische Leukämie (CLL). Internist 1996; 37: $1022-1029$

${ }^{6}$ Hochhaus A, Hehlmann R. Chronische myeloische Leukämie. Internist 1996; 37: $1013-1021$

${ }^{7}$ Hochhaus A, Hehlmann R. Chronische myeloproliferative Erkrankungen. In: Ostendorf PC, Seeberg S (Hrsg). Hämatologie Onkologie. München: Urban u. Schwarzenberg, 1997

${ }^{8}$ Kresbach H, Kerl H. Myeloproliferative Erkrankungen der Haut. In: Luger A, Gschnait F (Hrsg). Dermatologische Onkologie. Wien: Urban u. Schwarzenberg, 1983

${ }^{9}$ Kerl H, Kresbach H, Hödel ST. Klinische und histologische Kriterien zur Diagnose und Klassifikation der Leukämien der Haut. Hautarzt Suppl III 1978; 28: $97-101$
${ }^{10}$ Su WPD, Buechner SA, Chin-Yang L. Clinicopathologic correlations in leukemia cutis. J Am Acad Dermatol 1984; 11: 121 - 128

${ }^{11}$ Epstein E, MacEachern K. Dermatologic manifestations of the lymphoblastoma-leukemia group. Arch Intern Med 1937; 60: 867-875

12 Matutes E, Brito-Babapulle V, Swansburry J. Clinical and laboratory features of 78 cases of T-prolymphocytic leukemia. Blood 1991; 78: 3269-3274

${ }^{13}$ Kresbach H, Kerl H. Leukämische Hauterkrankungen. Hautarzt Suppl V 1981; 32: $232-235$

${ }^{14}$ Itoh K, Asai T, Sugiura Y. A case of acute myeloid leukemia with generalized leukemia cutis: Statistical analysis of skin involvement of autopsied leukemia in Japan. Rinshou Ketsueki 1978; 19: 1560 - 1568

${ }^{15}$ Saito H, Himori T, Sugawara K. Leukemia cutis: A case report and its incidence in Japan. Rinshou Ketsueki 1970; 11: $712-721$

16 Straus DJ, Mertelsmann R, Koziner B. The acute monocytic leukemias: Multidisciplinary studies in 45 patients. Medicine 1980; 59: 409-425

17 Tobelem G, Jacquillat C, Chastang C. Acute monoblastic leukemia: A clinical and biologic study of 74 cases. Blood 1980; 55: 71 - 76

${ }^{18}$ Meidenbauer N, Schlake G, Bross K. Symptomatische leukämische Infiltration der Lunge als Komplikation einer akuten myeloischen Leukämie. Dtsch med Wsch 1998; 123: 110-113

${ }^{19}$ Boggs DR, Wintrobe MM, Cartwright GE. The acute leukemias: Analysis of 322 cases and review of the literature. Medicine (Baltimore) 1962; 41: $163-225$

${ }^{20}$ Murphy WG, Fotheringham GH, Busuttil A. Skin lesions in chronic granulocytic leukemia: Treatment of a patient with topical nitrogen mustard. Cancer 1985; 55: 2630-2633

${ }^{21}$ Jänner M. Hämoblastosen der Haut. In: Korting GW (Hrsg). Dermatologie in Praxis und Klinik, Bd IV. Stuttgart: Thieme, 1981

${ }^{22}$ Baer MR, Barcos M, Farrel H. Acute myelogenous leukemia with leukemia cutis. Cancer 1989; 63: 2192-2200

${ }^{23}$ Hansen RM, Barnett J, Hanson G. Aleukemic leukemia cutis. Arch Dermatol 1986; 122 : $812-814$

${ }^{24}$ Horlick HP, Silvers DN, Knobler EH. Acute myelomonocytic leukemia presenting as a benign-appearing cutaneous eruption. Arch Dermatol 1990; 126: $653-656$

${ }^{25}$ Klein UE, Ude P. Monozytenleukämie mit ungewöhnlichem Erkrankungsablauf. Med Klin 1975; 70: 613-621

${ }^{26}$ Stawiski MA. Skin manifestations of leukemias and lymphomas. Cutis 1978; 21: 814-818

${ }^{27}$ Ratnam KV, Khor CJL, Su WPD. Leukemia cutis. Dermatol Clin 1994; 12: $419-431$

${ }^{28}$ Wohlenberg H, Griss P, Goos M. Zur Zytochemie von Hautinfiltraten bei myelomonozytärer Leukämie. DMW 1970; 95: 1439-1443

29 Bork K, Hoede N. Hautveränderungen bei malignen Erkrankungen. Internist 1983; 24: $321-328$

${ }^{30}$ Hubler WR, Netherton EW. Cutaneous manifestations of monocytic leukemia. Arch Dermatol 1947; 56: 70-89

${ }^{31}$ Zax RH, Kulp-Shorten CL, Callen JP. Leukemia cutis presenting as a scrotal ulcer. J Am Acad Dermatol 1989; 21: 410 - 413

32 Lynch MA, Ship II. Initial oral manifestations of leukemia. J Am Dent Assoc 1967; 75: 932 - 940

${ }^{33}$ Baden JT, Gammon WR. Leukemia cutis in acute myelomonocytic leukemia. Arch Dermatol 1987; 123: 88 -90

${ }^{34}$ Desch JK, Smoller BR. The spectrum of cutaneous disease in leukemias. J Cutan Pathol 1993; 20: 407-410

35 Wagner G, Ackermann H. Vasculitis allergica - retrospektive Analyse und Interpretation der Befunde von 88 Patienten. derm 2002; 8: $433-444$

${ }^{36}$ Cohen PR, Talpaz M, Kurzrock R. Malignancy - associated Sweet's syndrome: Review of the world literature. J Clin Oncol 1988; 6: 1887 1897

37 Caughman W, Stern R, Haynes H. Neutrophilic dermatosis of myeloproliferative disorders: Atypical forms of pyoderma gangrenosum and Sweet's syndrome associated with myeloproliferative disorders. J Am Acad Dermatol 1983; 9: 751 - 758

${ }^{38}$ Verhagen C, Stalpers LJA, de Pauw BE. Drug-induced skin reactions in patients with acute non-lymphocytic leukemia. Eur J Haematol 1987; 38: $225-230$

${ }^{39}$ Horn TD, Redd JV, Karp JE. Cutaneous eruptions of lymphocyte recovery. Arch Dermatol 1989; 125: $1512-1517$ 